\title{
Inhibition of Lysyl Oxidases Impairs Migration and Angiogenic Properties of Tumor-Associated Pericytes
}

\author{
Aline Lopes Ribeiro, Carolini Kaid, Patrícia B. G. Silva, Beatriz A. Cortez, and \\ Oswaldo Keith Okamoto
}

Centro de Pesquisa sobre o Genoma Humano e Células-Tronco, Departamento de Genética e Biologia Evolutiva, Instituto de Biociências, Universidade de São Paulo, Rua do Matão 277, Cidade Universitária, 05508-090 São Paulo, SP, Brazil

Correspondence should be addressed to Oswaldo Keith Okamoto; keith.okamoto@usp.br

Received 18 October 2016; Accepted 5 March 2017; Published 3 May 2017

Academic Editor: Dominique Bonnet

Copyright ( 2017 Aline Lopes Ribeiro et al. This is an open access article distributed under the Creative Commons Attribution License, which permits unrestricted use, distribution, and reproduction in any medium, provided the original work is properly cited.

\begin{abstract}
Pericytes are important cellular components of the tumor microenviroment with established roles in angiogenesis and metastasis. These two cancer hallmarks are modulated by enzymes of the LOX family, but thus far, information about LOX relevance in tumor-associated pericytes is lacking. Here, we performed a comparative characterization of normal and tumoral pericytes and report for the first time the modulatory effects of LOX enzymes on activated pericyte properties. Tumoral pericytes isolated from childhood ependymoma and neuroblastoma specimens displayed angiogenic properties in vitro and expressed typical markers, including CD146, NG2, and PDGFR $\beta$. Expression of all LOX family members could be detected in both normal and tumor-associated pericytes. In most pericyte samples, LOXL3 was the family member displaying the highest transcript levels. Inhibition of LOX/LOXL activity with the inhibitor $\beta$-aminopropionitrile ( $\beta \mathrm{APN})$ significantly reduced migration of pericytes, while proliferation rates were kept unaltered. Formation of tube-like structures in vitro by pericytes was also significantly impaired upon inhibition of LOX/LOXL activity with $\beta A P N$, which induced more prominent effects in tumor-associated pericytes. These findings reveal a novel involvement of the LOX family of enzymes in migration and angiogenic properties of pericytes, with implications in tumor development and in therapeutic targeting tumor microenvironment constituents.
\end{abstract}

\section{Introduction}

It has been increasingly recognized that the multistep process of tumor development is driven not only by genetic modifications but also by nonmalignant stromal cells within the tumor microenvironment (TME), such as immune cells, endothelial cells, pericytes, and fibroblasts $[1,2]$. In addition, extracellular matrix, growth factors, chemokines, and other TME components provide a critical communication network between stromal and tumor cells, impacting both tumor progression and efficacy of anticancer therapies [3-5].

One important, albeit less studied, cellular component of the TME is the pericyte population [6]. Pericytes are present along the microvessel walls where they are important for vasculature remodeling, maturation and stabilization, regulation of blood flow, and blood vessel permeability. Multipotential differentiation capacity $[7,8]$ as well as immunomodulatory properties, particularly for central nervous system (CNS) pericytes, has also been reported for these cells $[9,10]$.

Pericytes are closely attached to endothelial cells providing mechanical and physiological support [10]. The endothelial cell-pericyte communication is essential for regulating proliferation, migration, and differentiation of both cell types, which act in concert to coordinate angiogenesis [11]. During the normal vascular sprouting process, pericytes become activated, undergoing phenotypical and functional modifications. Pericyte activation involves enhanced cell migration, proliferation, and secretion of molecules such as proteases, which degrade the basement membrane and modulate the extracellular matrix structure $[12,13]$.

In cancer, the tumor vascular structure does not achieve proper maturation and, as a consequence, has several abnormalities including irregular pericyte coverage [14]. Furthermore, tumor pericytes are loosely attached to the 
TABLE 1: Expression of cell surface markers in pericytes derived from normal tissue and pediatric nervous system tumors.

\begin{tabular}{|c|c|c|c|c|}
\hline \multirow{2}{*}{ Cell surface marker } & \multicolumn{2}{|c|}{ Normal pericytes } & \multicolumn{2}{|c|}{ Tumor-associated pericytes } \\
\hline & NP-Ad $(\%)^{*}$ & $\mathrm{NP}-\mathrm{Mu}(\%)^{*}$ & TP-Nbl $(\%)^{*}$ & TP-Epn $(\%)^{*}$ \\
\hline CD146 & 95,0 & 65,5 & 0,4 & 46,4 \\
\hline NG2 & 99,4 & 94,1 & 22,7 & 99,6 \\
\hline PDGFR $\beta$ & 93,1 & 70,3 & 63,0 & 59,6 \\
\hline CD31 & 0,3 & 0,8 & 1,0 & 10,2 \\
\hline $\mathrm{CD} 45$ & 0,8 & 0,2 & 0,1 & 0,1 \\
\hline CD105 & 5,0 & 11,7 & 2,2 & 7,2 \\
\hline CD90 & 34,1 & 60,6 & 24,6 & 99,7 \\
\hline $\mathrm{CD} 73$ & 100,0 & 100,0 & 100,0 & 100,0 \\
\hline
\end{tabular}

${ }^{*}$ Percentage of positive cells for each surface marker, within a given pericyte culture, as determined by flow cytometric analysis. NP-Ad: normal pericytes from adipose tissue; NP-Mu: normal pericytes from muscle tissue; TP-Nbl: tumoral pericytes from neuroblastoma; TP-Epn: tumoral pericytes from ependymoma.

endothelium and present aberrant cytoplasmic projections invading the tumor parenchyma [13]. It is largely unknown whether mechanisms involved in pericyte activation are affected during tumor angiogenesis.

Recent studies have implicated lysyl oxidases (LOX) in vascular remodeling during angiogenesis [15-17]. This is an emerging role for the LOX family of secreted copperdependent amine oxidases, whose primary function is the covalent crosslinking of collagens and/or elastin in the extracellular matrix, maintaining tissue strength and structural integrity [18]. The LOX family is comprised of five members: LOX and LOX-like 1-4 (LOXL1-4), which also have important effects on cell senescence, differentiation, and migration [19]. Due to their role in extracellular remodeling, deregulation of enzymes of the LOX family is involved in different diseases [20].

Several studies have extensively demonstrated expression of distinct LOX family members in various types of tumor cells, implicating their functions in tumor progression and metastasis [21, 22]. Despite the growing evidences of LOX contribution to the establishment of a protumorigenic microenvironment [23], there is very few information available about tumor stroma-derived $\operatorname{LOX}[24,25]$. Thus far, expression of LOX family members in pericytes and LOX influence in pericyte protumorigenic properties have not been examined.

Here, we performed a comparative characterization of normal and tumor-associated pericytes and report for the first time the modulatory effects of LOX enzymes on activated pericyte properties directly implicated in tumor progression, namely migration, proliferation, and angiogenic activity.

\section{Materials and Methods}

2.1. Primary Cell Cultures. Pericyte samples, NP-Ad (normal pericytes from adipose tissue) and NP-Mu (normal pericytes from muscle tissue), and mesenchymal stem cells (MSCs), PLAJ3 (adipose tissue) and UC3 (umbilical cord), were obtained, isolated, and cultivated as previously described $[26,27]$. Primary cultures of tumor-derived pericytes, TP$\mathrm{Nbl}$ (tumoral pericytes from neuroblastoma) and TP-Epn (tumoral pericytes from ependymoma), were obtained in collaboration with Dr. Hamilton Matushita from the Faculdade de Medicina, Universidade de São Paulo. Isolation and culture methods were conducted as described by Valadares et al. [26]. All procedures were approved by the institutional review board (CEP-IB number 121/2011), and informed consent was obtained from all donors. Human umbilical vein endothelial cells (HUVEC) were purchased directly from ATCC (Manassas, VA, USA) and cultivated under the same conditions as pericytes.

2.2. Flow Cytometry. Cells were labeled for 40 minutes at $4^{\circ} \mathrm{C}$ with the following antibodies: CD146-APC (1:100, R\&D Systems, Minneapolis, MN, USA); NG2-PE (1:100, R\&D Systems); PDGFR $\beta$-PE ( $1: 100$, R\&D Systems); CD31-FITC (1:100, BioLegend, San Diego, CA, USA); CD45-APC (1:100, BioLegend); CD105-FITC (1:100, BioLegend); CD90-PE ( $1: 100$, BD Biosciences, San Jose, CA, USA); and CD73-PE (1:100, BioLegend). Analyses were performed using Guava EasyCyte 5HT ${ }^{\mathrm{TM}}$ Flow Cytometer and GuavaSoft 2.1 software (Millipore, Billerica, MA, USA).

2.3. In Vitro Angiogenesis Assay and Time-Lapse Analysis. Corning ${ }^{\circledR}$ Matrigel ${ }^{\circledR}$ Basement Membrane Matrix (Corning, Corning, NY, USA) was transferred to a precooled 96-well plate. The plates were incubated at $37^{\circ} \mathrm{C}$ for 30 minutes. Single cells $\left(2 \times 10^{4}\right.$ cells $)$ were added to each well and incubated in a humidified $5 \% \mathrm{CO}_{2}$ atmosphere at $37^{\circ} \mathrm{C}$. After 2 hours, the tubular networks were examined under the microscope and time-lapse captures were taken every 30 minutes for 24 hours using Incell Analyzer 2200 (GE Healthcare, Little Chalfont, UK). The obtained images were analyzed, and the total amount of lumens formed (considered as internal regions surrounded by cell cords), in three wells per experimental condition, was entirely counted using ImageJ software.

2.4. Expression of LOX Family Members. Total RNA was extracted and reverse transcribed as previously described [28]. Reactions of qRT-PCR were performed in triplicate using Power SYBR Green Master Mix (Life Technologies, Carlsbad, CA, USA) on Applied Biosystem 7500 Real-Time PCR System (Life Technologies). Expression of the housekeeping gene TBP was used as endogenous control. Quantitative analyses were based on a relative quantification curve, using MSC UC3 as a positive control sample. Primer 


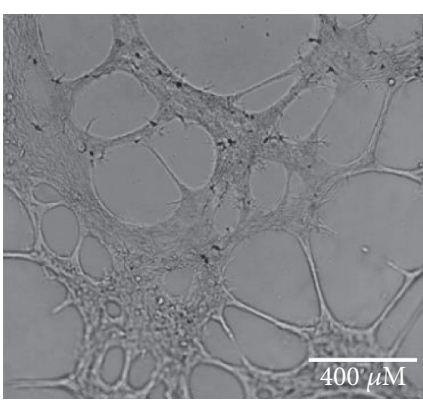

(a)

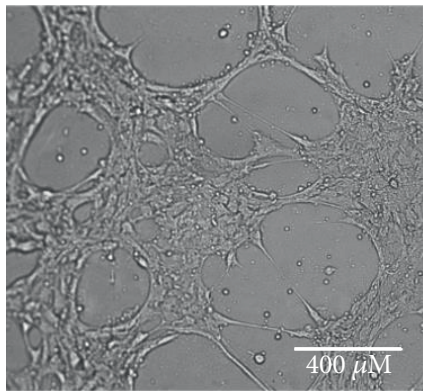

(c)

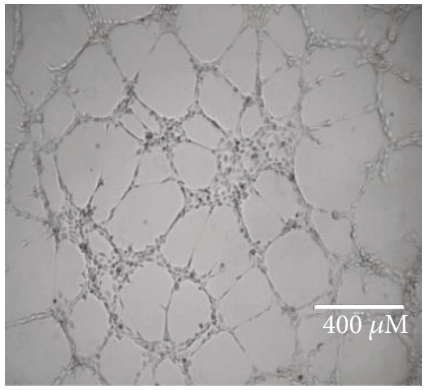

(e)

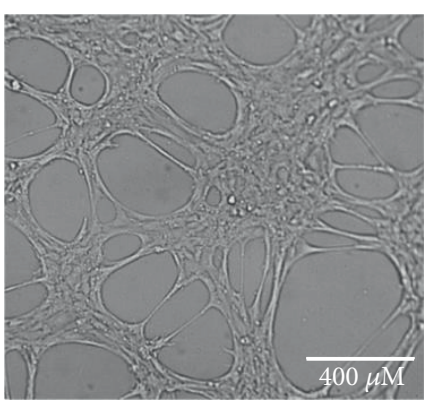

(b)

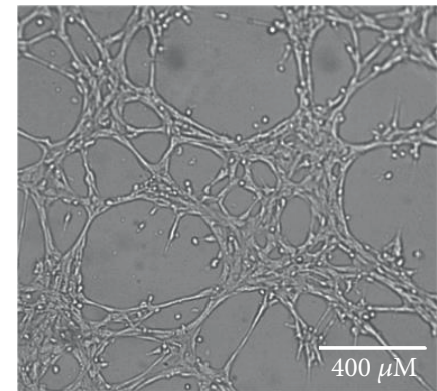

(d)

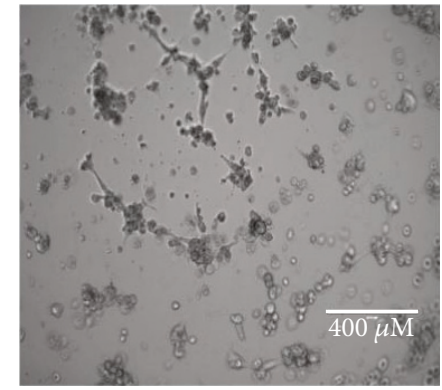

$(\mathrm{f})$

Figure 1: Angiogenic properties of pericytes. Both normal (NP-Ad, NP-Mu) and tumor-associated pericytes (TP-Nbl, TP-Epn) were capable of forming tube-like structures in vitro, generating interconnected networks similar to those generated by endothelial cells (HUVEC). Mesenchymal stem cells (MSCs) do not share this propriety and, therefore, were not able to generate such tube-like structures under the same experimental conditions. (a) NP-Ad; (b) NP-Mu; (c) TP-Nbl; (d) TP-Epn; (e) HUVEC; (f) MSC.

sequences: TBP-For: GAGCTGTGATGTGAAGTTT CC; TBP-Rev: TCTGGGTTTGATCATTCTGTAG; LOX-For: AAGAGTGAAAAACCAAGG GACA; LOX-Rev: TGGTAG CCATAGTCACAGGATG; LOXL1-For: TG GTAGC CATA GTCACAGGATG; LOXL1-Rev: AAGAGTGAAAAACCAA GGGACA; LOXL2-For: TCGAGGTTGCAGAATCCGATT; LOXL2-Rev: TTCCGTCTCTT CGCTGAAGGA; LOXL3For: CGGATGTGAAGCCAGGAAAC; LOXL3-Rev: AG GC ATCACCAATGTGGCA; LOXL4-For: GGCAGAGTCAGA TTTCTCCAACA; LOXL4-Rev: GAGTTCTGCATTGGCTG GGTAT. For detection of LOX/LOXL protein levels, total proteins were extracted with RIPA buffer (Sigma-Aldrich, St. Louis, MO, USA) following the manufacturer's recommendations and submitted to Western blotting standard protocol. Proteins were transferred to nitrocellulose membrane (GE Healthcare). After 1 hour of blocking, membranes were incubated overnight with the following primary antibodies: LOX (Abcam, Cambridge, UK), LOXL3 (Abcam), and GAPDH (Abcam). Blots were incubated for 1 hour with secondary antibody (anti-rabbit HRP-linked) and developed using ECL Chemiluminescence Detection System (GE Healthcare).

2.5. Inhibition of LOX/LOXL Activity. $\beta$-Aminopropionitrile $(\beta \mathrm{APN})$ is commonly used to inhibit the activity of LOX enzymes since it acts as an active site inhibitor [22, 29]. Cells were pretreated with $1 \mu \mathrm{M} \beta \mathrm{APN}$ for 24 hours before the assays. Additionally, $\beta$ APN was also added to the culture medium during all assay procedures at the same final concentration. The inhibition efficacy was verified by measuring $\mathrm{H}_{2} \mathrm{O}_{2}$ after reaction with DCFH-DA (SigmaAldrich) in a flow cytometer, as described by Eruslanov and Kusmartsev [30].

2.6. Cell Viability Assay. Cell viability was assessed by the MTT assay (Sigma-Aldrich). Briefly, cells at a density of $2 \times 10^{4} / \mathrm{mL}$ were seeded into a 24 -well plate and incubated at $37^{\circ} \mathrm{C}$ with $5 \% \mathrm{CO}_{2}$ humidified atmosphere for 24 hours. 


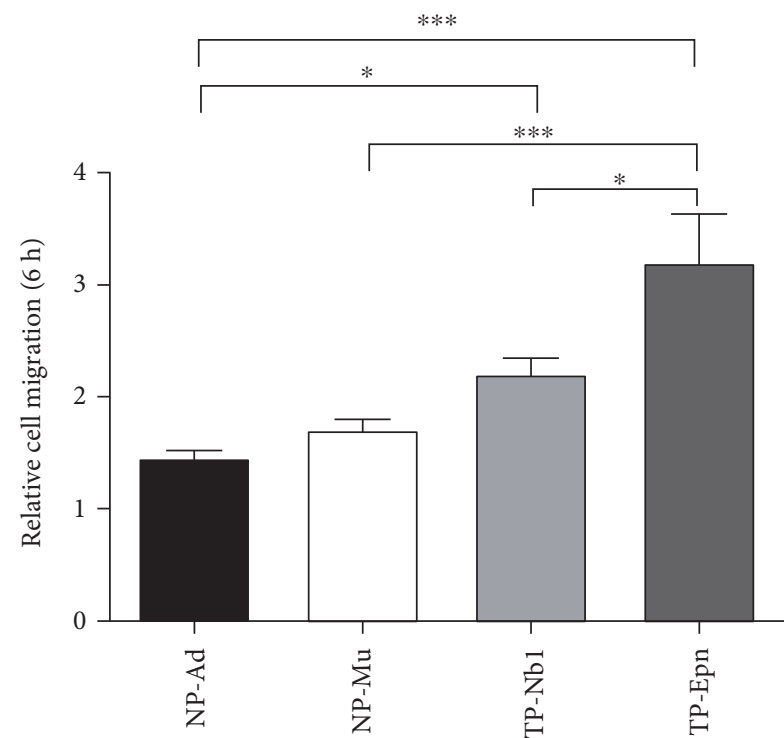

(a)

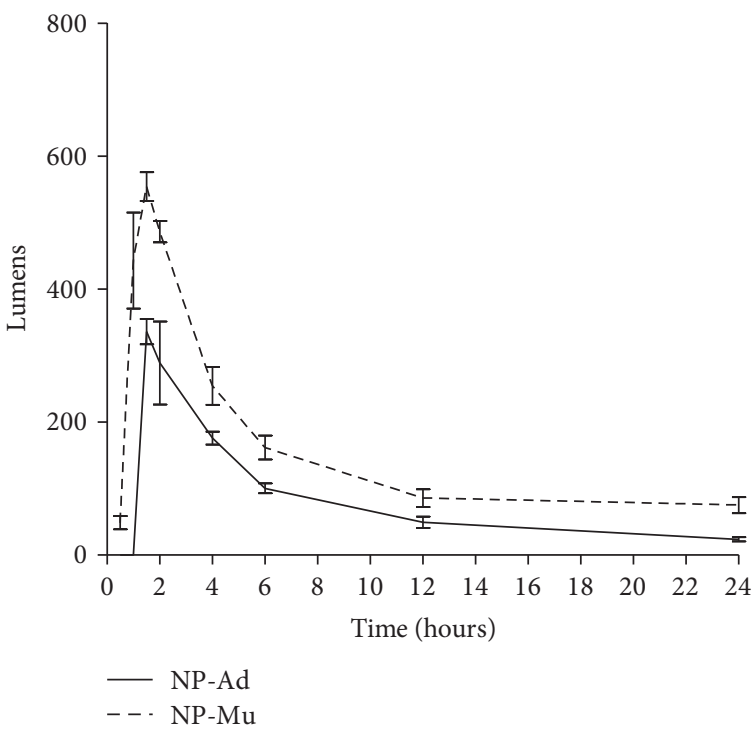

(c)

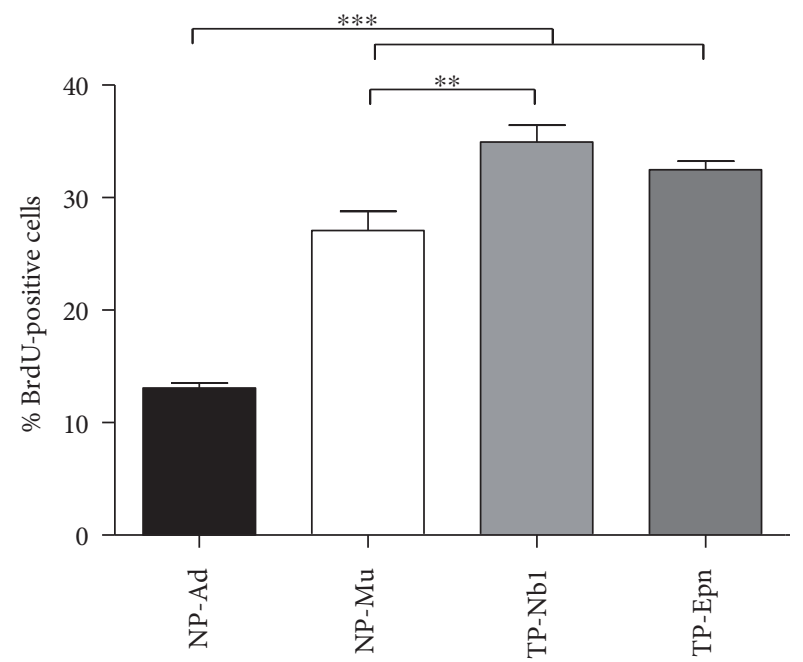

(b)

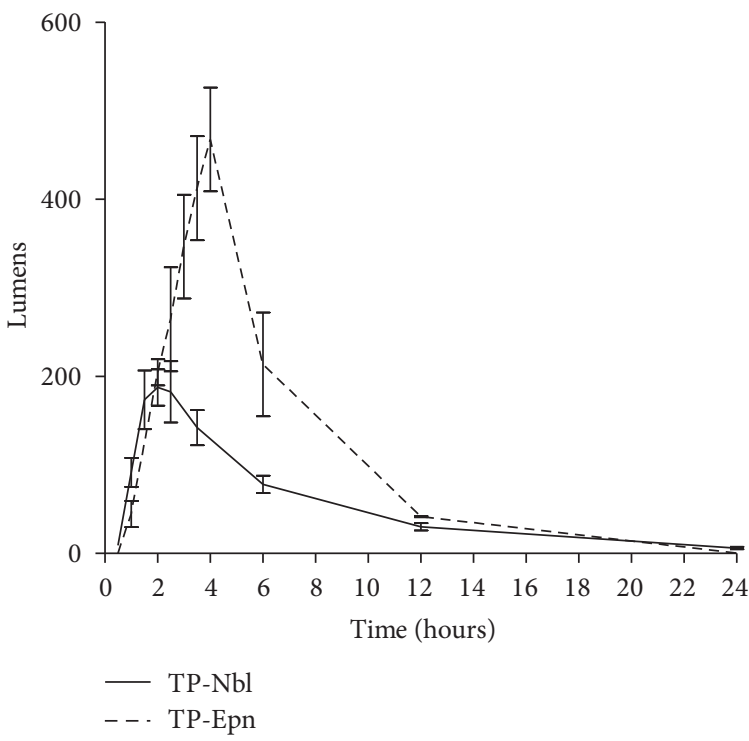

(d)

Figure 2: Activated properties of normal and tumor-associated pericytes. (a) Cell migration was calculated after $6 \mathrm{~h}$ in the presence of chemical stimulus, relative to basal cell migration without chemotaxis. (b) Cell proliferation based on BrdU incorporation after $24 \mathrm{~h}$ in culture. Kinetics of tube-like formation by (c) normal pericytes and (d) tumor-associated pericytes, under angiogenic conditions in vitro. Normal pericyte samples: NP-Ad and NP-Mu. Tumor-associated pericyte samples: TP-Nbl and TP-Epn. Significance level: ${ }^{*} P<0.05$, ${ }^{* *} P<0.01$, and ${ }^{* * *} P<0.001$.

Cells were treated for 24,48 , and 72 hours with fresh medium containing $1 \mu \mathrm{M} \beta \mathrm{APN}$ or vehicle (control). After treatment periods, MTT solution was added at concentration of $166.67 \mu \mathrm{g} / \mathrm{mL}$ and incubated for 4 hours at $37^{\circ} \mathrm{C}$. The supernatant was discarded and formazan crystals were dissolved with DMSO. The optical density was measured at $550 \mathrm{~nm}$ using Epoch Microplate Spectrophotometer (BioTek, Winooski, VT, USA).

2.7. Cell Migration Assay. Cell migration assay was performed using modified Boyden chambers as described by Chen [31]. Briefly, $1 \times 10^{5}$ cells were plated at the upper compartment of a Transwell ${ }^{\circledR}$ with $8.0 \mu \mathrm{m}$ pore polyester membrane insert (Corning) and medium with $10 \%$ FBS was placed at the inferior compartment to act as a chemoattractive solution. Following an incubation period (3 hours for tumor pericytes and 12 hours for normal tissue pericytes), the cells that migrated through the membrane were fixed and stained with $1 \%$ of toluidine blue and $1 \%$ borax solution. Stained cells were lysed with $1 \%$ SDS, and optical density was measured at $626 \mathrm{~nm}$.

2.8. EdU Incorporation Assay. Cells in the $\mathrm{S}$ phase of cell cycle were determined by EdU incorporation assay using 


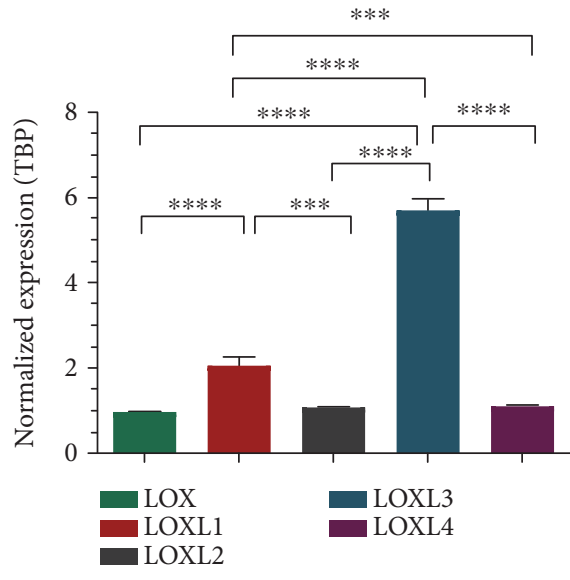

(a)

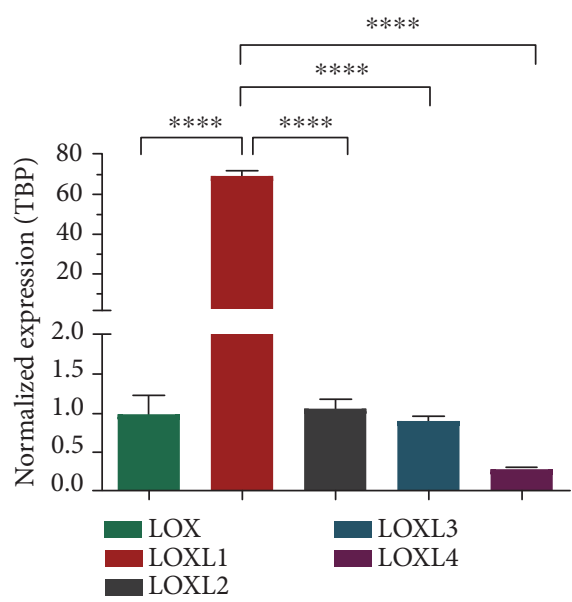

(c)

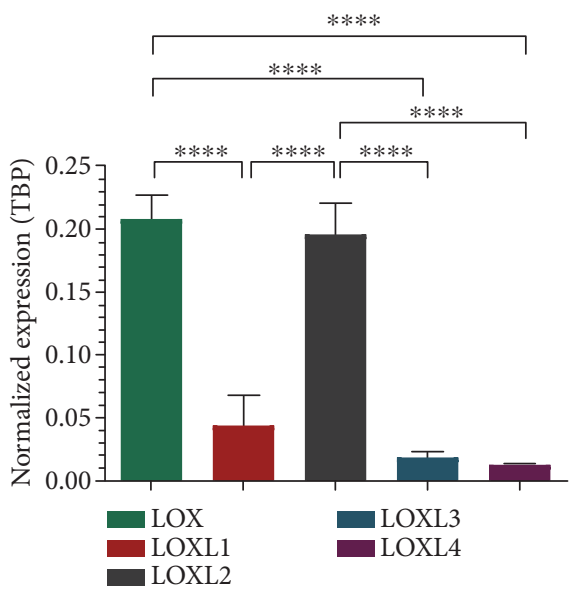

(e)

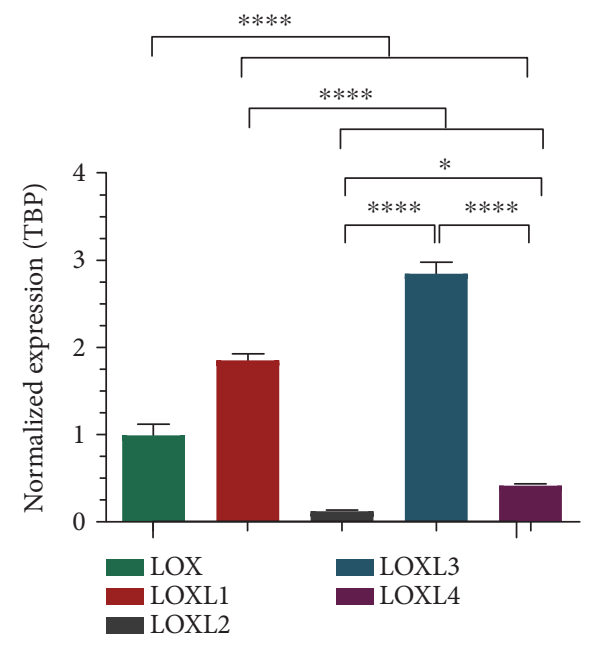

(b)

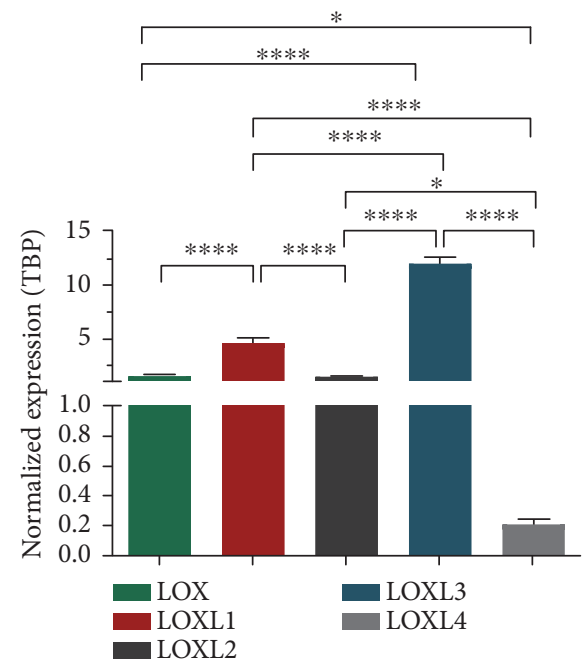

(d)

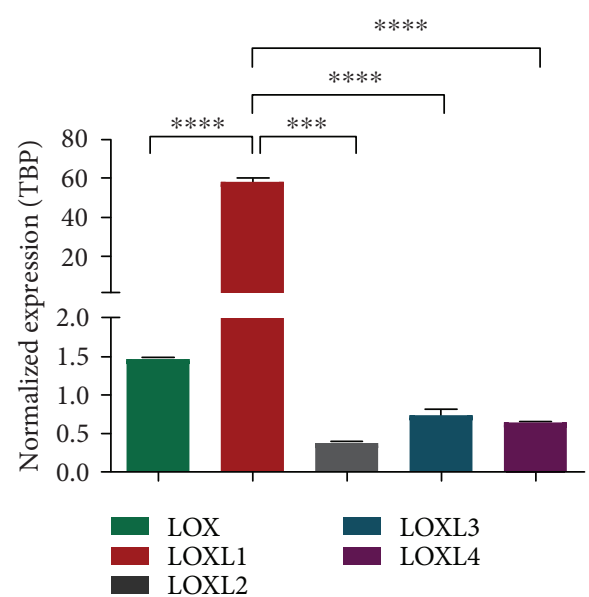

(f)

FIGURE 3: Comparative analysis of LOX and LOXL1-4 expression profiles in normal and tumor-associated pericytes. Gene expression was assessed by quantitative real-time PCR, using TBP as endogenous control. Normal pericyte samples: NP-Ad and NP-Mu. Tumorassociated pericyte samples: TP-Nbl and TP-Epn. Endothelial cells (HUVEC) and mesenchymal stem cells (MSCs) were also included in the analysis as control samples. (a) NP-Ad; (b) NP-Mu; (c) TP-Nbl; (d) TP-Epn; (e) HUVEC; (f) MSC. Significance level: ${ }^{*} P<0.05$, ${ }^{* *} P<0.01,{ }^{* * *} P<0.001$, and ${ }^{* * * *} P<0.0001$. 
Click-It EdU Alexa Fluor 488 Imaging Kit (Life Technologies) and following the manufacturer's recommendation. EdU $(10 \mu \mathrm{M})$ incorporation time was 2 hours for all pericyte cultures.

2.9. Statistical Analysis. Data were analyzed by ANOVA followed by the Bonferroni post hoc test or unpaired Student's $t$-test (GraphPad Prism 5 software, GraphPad Software, La Jolla, CA, USA) as indicated in the figure legends. All experiments were performed at least in triplicate, and three independent experiments were carried out. Results are presented as mean $\pm \mathrm{SD}$.

\section{Results}

3.1. Abnormal Tumoral Pericyte Properties. As indicated in Table 1, tumor-associated pericytes displayed a rather heterogeneous pattern of expression of the typical pericyte markers, CD146, NG2, and PDGFR $\beta$, compared with normal pericytes. However, as expected, both normal and tumor-associated pericyte populations were mainly negative for the expression of endothelial and hematopoietic cell markers and did not display the combined expression of CD90, CD73, and CD105, a required characteristic of mesenchymal stromal cells (MSCs) [32]. Further functional tube formation assays confirmed that all normal and tumorassociated pericyte cultures were comprised by cells capable of generating capillary-like structures in vitro (Figures 1(a), 1(b), 1(c), and 1(d)), a characteristic property of pericytes and endothelial cells, but not of MSCs (Figures 1(e) and 1(f)).

A more detailed analysis revealed that, under the same experimental conditions, tumor-associated pericytes presented significantly higher migration and proliferation rates compared with normal pericytes (Figures 2(a) and 2(b)). When comparing the kinetics of tube formation in vitro (Figures 2(c) and 2(d)), tumor-associated pericytes either generated a lower amount of total capillary-like structures or took a longer time to attain the maximum amount of such structures than normal pericytes, indicating an abnormal in vitro behavior.

3.2. Expression Profile of Lysyl Oxidases in Pericytes. Since no previous studies were available regarding expression of LOX family members in pericytes, a LOX/LOXL expression profiling was carried out in the two normal tissuederived pericytes and two tumor-associated pericytes and compared with the expression levels detected in MSCs and endothelial cells, which are known to be positive for LOX/LOXL expression.

At the transcriptional level, a concordant LOX/LOXL expression pattern was detected in the two normal tissuederived pericytes analyzed. Expression of all LOX family members could be detected in these cells, with LOXL3 exhibiting the highest expression levels (Figures 3(a) and 3(b)). A similar LOX/LOXL expression pattern was detected in the pericyte sample derived from ependymoma, but not in the other pericyte sample derived from neuroblastoma (Figures 3(c) and 3(d)). In the latter case, LOXL1 displayed

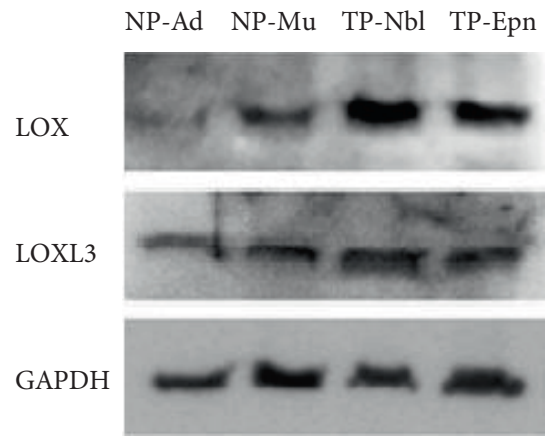

FIGURE 4: LOX and LOXL3 protein levels in normal and tumorassociated pericytes. Representative Western blots for each protein are shown. Normal pericyte samples: NP-Ad and NP-Mu. Tumorassociated pericyte samples: TP-Nbl and TP-Epn.

the highest expression levels, such as the one exhibited by MSCs (Figure 3(f)).

Nonetheless, the LOX/LOXL expression profile detected in normal and tumor-associated pericytes differed from the one exhibited by endothelial cells, in which LOX and LOXL2 are the predominantly expressed family members (Figure 3(e)).

Expression of LOX and LOXL3 in pericytes was also confirmed at the protein level. Higher LOX levels were detected in tumor-associated pericytes compared with normal pericytes (Figure 4).

\subsection{Inhibition of Lysyl Oxidases Affects Protumorigenic} Properties of Pericytes. The effects of LOX/LOXL on activated pericyte properties were investigated using the specific inhibitor $\beta \mathrm{APN}$, under experimental conditions ensuring significant inhibition of enzymatic activity, without affecting cell viability (Supplementary Figure available online at https://doi.org/10.1155/2017/4972078).

Upon inhibition of LOX/LOXL activity, a significant reduction in migration of both normal and tumor-associated pericytes was detected. Cell proliferation rates, however, were not altered under the same experimental conditions (Figures 5(a) and 5(b)).

Tube formation dynamics in vitro by pericytes was also affected upon inhibition of LOX/LOXL activity (Figure 6). Compared with control conditions, there was a significant reduction in the total number of lumens formed and, for some pericyte cultures, the kinetic curves were also slightly dislocated. The most prominent effects were observed in cultures of tumor-associated pericytes, where the time for reaching the maximum amount of capillary-like structures was longer after inhibition of LOX/LOXL activity.

Altogether, these results indicate that blocking activity of LOX family members may influence pericyte properties that are essential to their protumorigenic roles within the TME.

\section{Discussion}

Understanding pericyte properties is essential given the effects that these cells may exert within the TME [33-37]. Despite the lack of consensus in the current literature about 

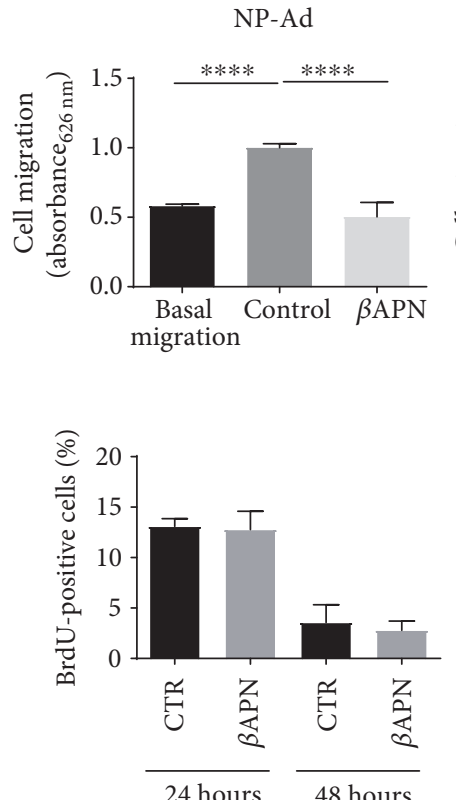
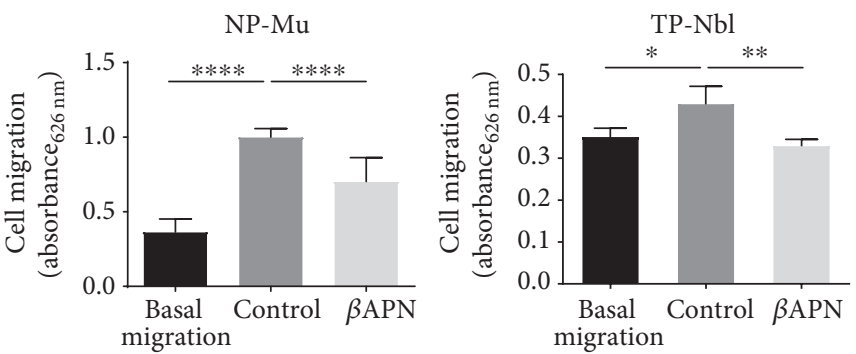

(a)

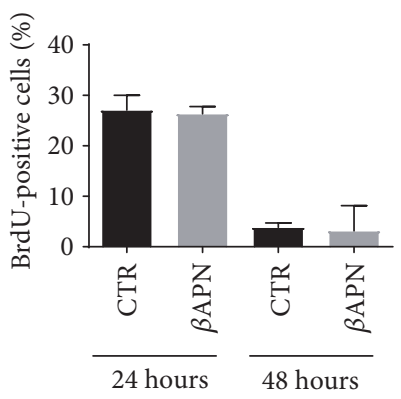

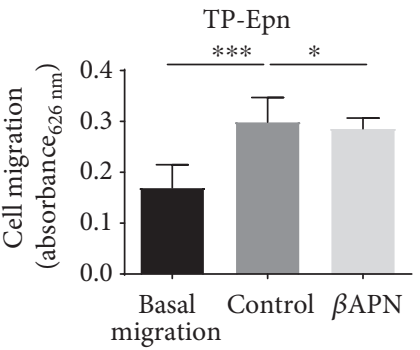

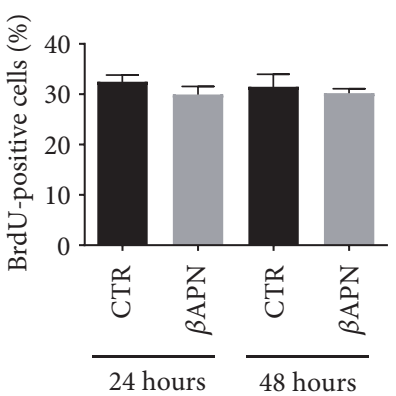

(b)

FIGURE 5: Effects of $\beta$ APN on migration and proliferation of normal and tumor-associated pericytes. (a) Cell migration measured after $24 \mathrm{~h}$ treatment with $1 \mu \mathrm{M} \beta \mathrm{APN}$. (b) Cell proliferation based on BrdU incorporation after $24 \mathrm{~h}$ and $48 \mathrm{~h}$ treatment with $1 \mu \mathrm{M} \beta \mathrm{APN}$. Normal pericyte samples: NP-Ad and NP-Mu. Tumor-associated pericyte samples: TP-Nbl and TP-Epn. Significance level: ${ }^{*} P<0.05,{ }^{* *} P<0.01$, ${ }^{* * *} P<0.001$, and ${ }^{* * * *} P<0.0001$.

definite criteria for pericyte identification, the selected normal and tumor-derived pericytes used in this study displayed in vitro angiogenic properties and expression of cellular markers typically reported for this type of cell [38]. Notably, expression of CD146, NG2, and PDGFR $\beta$ was somewhat reduced in the population of tumor-associated pericytes, in agreement with previous studies reporting variable expression of some of these markers in pathological conditions. In perivascular soft tissue tumors, a reduced expression of $\alpha$ SMA, CD146, and PDGFR $\beta$ in pericytes from malignant glomus tumors has been implicated in partial loss of pericytic differentiation [39]. In brain tumors, low expression of the NG2 proteoglycan in pericytes has been reported to affect their interaction with endothelial cells and disturb tumor vasculogenesis [40].

Within the CNS, pericytes play key physiological roles with effects in brain angioarchitecture, blood-brain barrier, and neuroinflammation [41-43]. Pericytes may also affect tumor malignancy and clinical prognosis, particularly in CNS tumors that are highly vascularized $[44,45]$. In the present study, the increased migration and proliferation rates observed for tumor-associated pericytes compared with normal pericytes are consistent with the TME context. Tumor stromal cells, including fibroblasts and endothelial cells, are known to be constantly activated, exhibiting enhanced migration and proliferative behavior [46, 47]. Mechanisms underlying tumoral pericyte activation are, however, still elusive.

A growing body of evidences indicates that processes relevant to tumor progression, such as angiogenesis and metastasis, are modulated by enzymes of the LOX family
$[21,23,48]$. Although these two processes involve interaction with the tumor stroma, thus far, very little is known about LOX expression in cellular components of the TME, and data on LOX expression in pericytes is lacking. Here, both normal and tumor-derived pericytes were found to express all members of the LOX family of enzymes. At the transcript level, the pattern of LOX/LOXL expression observed was very similar among the pericyte samples, the exception being the neuroblastoma-derived pericytes in which LOXL1 was more highly expressed than the other family members.

Apart from its role in extracellular matrix modulation and implications in pathogenesis $[49,50]$, no other functions are known for LOXL3, the family member that displayed the highest expression level in most pericyte samples. On the other hand, expression of LOX and LOXL2 has been frequently correlated with all stages of tumorigenesis. Secretion of LOXL 2 by both stromal and tumor cells, for instance, is involved in fibroblast activation within the TME [51, 52]. Regarding LOX/LOXL expression in tumor stromal cells, a recent study with a mouse model of mammary tumor progression showed that LOX expressed by carcinomaassociated fibroblasts stimulates tumor cell intravasation into the vasculature and ensuing metastasis [23].

The significant inhibition of migration and angiogenic properties of pericytes detected upon inhibition of LOX/ LOXL activity with the inhibitor $\beta A P N$ is in agreement with a previous study reporting inhibition of tumor angiogenesis by $\beta$ APN treatment in vivo [53]. Since knockdown of $L O X$ and LOXL2 has also been reported to inhibit motility and network-forming ability of endothelial cells [49], expression of LOX/LOXL in both pericytes and endothelial cells is likely 
Normal pericytes

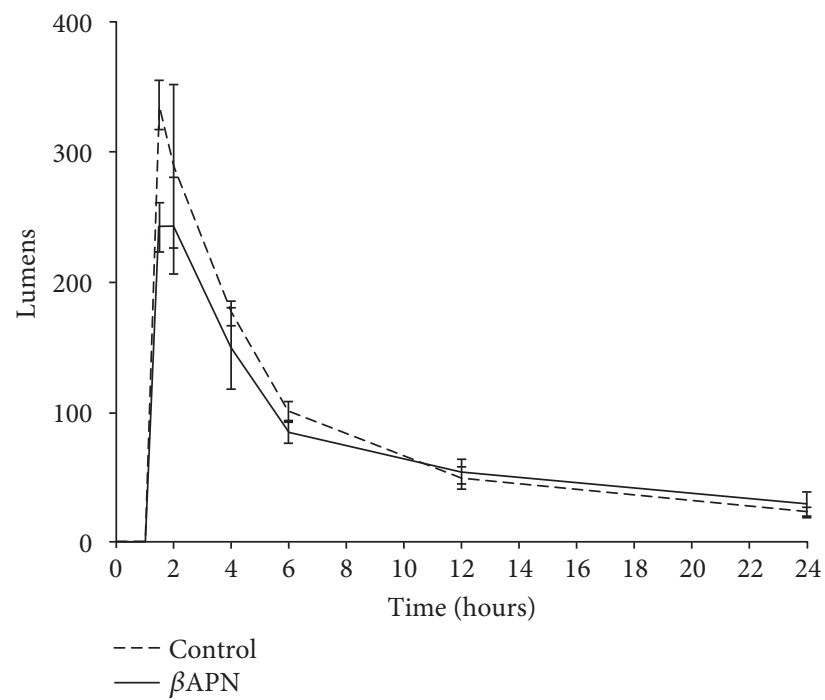

(a)

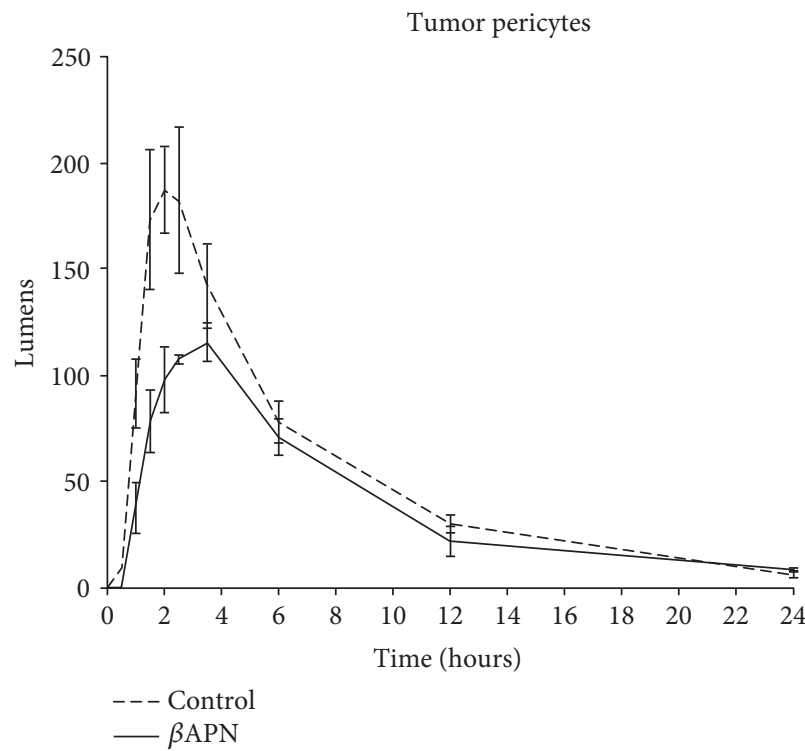

(c)
Normal pericytes

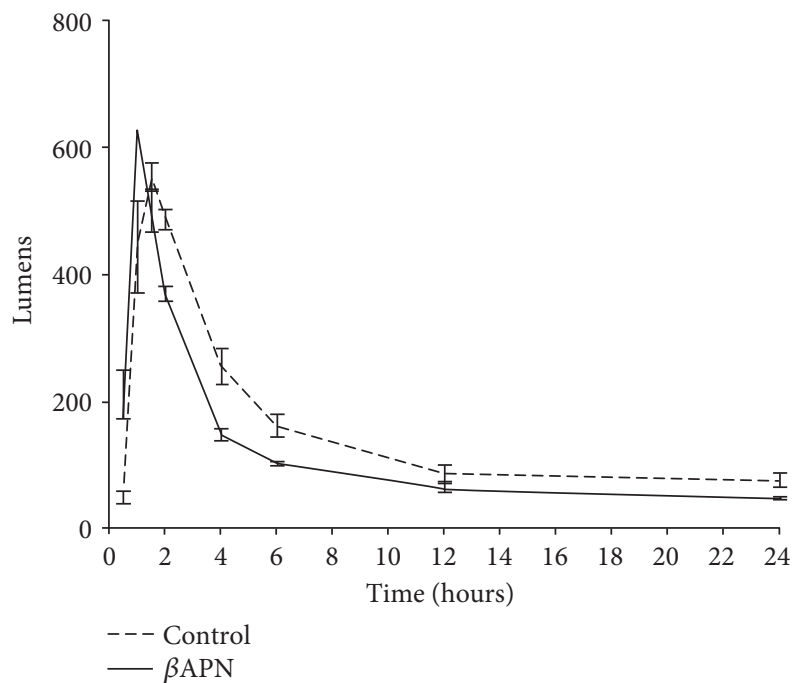

(b)

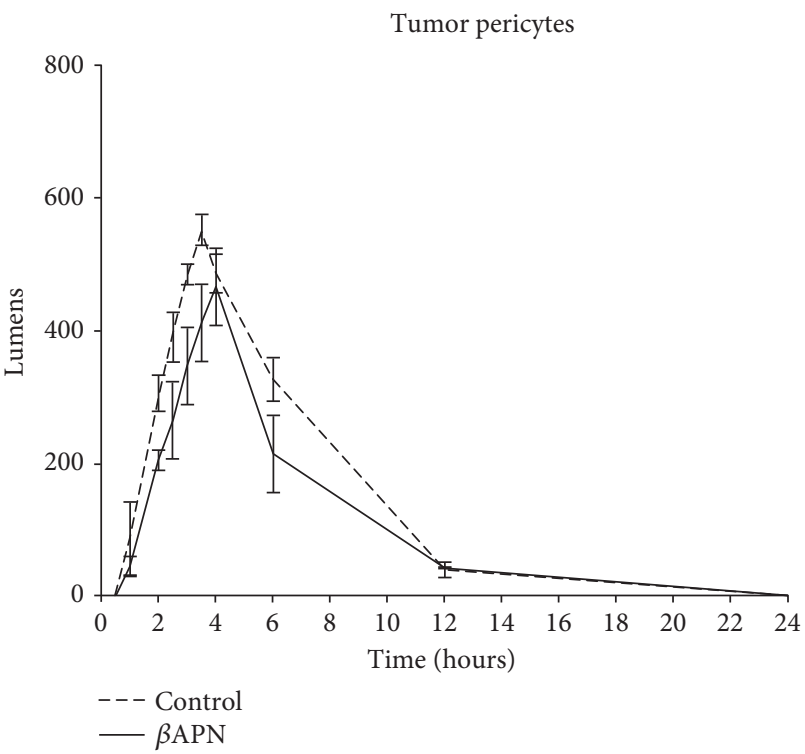

(d)

Figure 6: Effects of $\beta$ APN on angiogenic properties of normal and tumor-associated pericytes. The kinetics of tube-like formation by pericytes was assessed for 24 hours under treatment with $1 \mu \mathrm{M} \beta \mathrm{APN}$ in vitro. Normal pericyte samples: NP-Ad and NP-Mu. Tumorassociated pericyte samples: TP-Nbl and TP-Epn. (a) NP-Ad; (b) NP-Mu; (c) TP-Nbl; (d) TP-Epn.

indispensable for proper angiogenesis. Interestingly, in the in vitro angiogenesis assays, the inhibitory effects of $\beta$ APN treatment were more pronounced in tumor-associated pericytes than in their normal counterparts, given that not only a lower amount of total tube-like structures was observed but also a longer time to achieve the peak of such structures. This result is in concert with the higher LOX protein levels detected in tumor-associated pericytes, encouraging preclinical studies addressing whether antiangiogenic approaches based on LOX/LOXL inhibitors may be designed to be preferentially harming to pericytes associated with the tumor vasculature.

\section{Conclusions}

In summary, our findings reveal a novel involvement of LOX/LOXL family of enzymes in migration and angiogenic properties of both normal and tumor-associated pericytes, supporting the exploration of LOX/LOXL inhibitors to target activation of pericytes within the TME.

\section{Conflicts of Interest}

The authors disclose no potential conflicts of interest. 


\section{Acknowledgments}

This study was supported by funds from the Fundação de Amparo à Pesquisa do Estado de São Paulo (FAPESP), grant FAPESP-CEPID (2013/08028-1); funds from the Conselho Nacional de Desenvolvimento Científico e Tecnológico (CNPq), Grants CNPq (307392/2014-7; 444722/2014-9) and INCT-CETGEN (573633/2008-8); and funds from the Financiadora de Estudos e Projetos (FINEP), Grant FINEP-CTC (0108057900). The authors thank the Core Facility for Scientific Research-University of São Paulo (CEFAP-USP/CONFOCAL), for the InCell Analyzer 2200 GE support analysis.

\section{References}

[1] F. Chen, X. Zhuang, L. Lin et al., "New horizons in tumor microenvironment biology: challenges and opportunities," BMC Medicine, vol. 13, p. 45, 2015.

[2] D. Hanahan and R. A. Weinberg, "Hallmarks of cancer: the next generation," Cell, vol. 144, no. 5, pp. 646-674, 2011.

[3] H. Bolouri, "Network dynamics in the tumor microenvironment," Seminars in Cancer Biology, vol. 30, no. 2, pp. 52-59, 2015.

[4] F. Klemm and J. A. Joyce, "Microenvironmental regulation of therapeutic response in cancer," Trends in Cell Biology, vol. 25, no. 4, pp. 198-213, 2015.

[5] H. Ungefroren, S. Sebens, D. Seidl, H. Lehnert, and R. Hass, "Interaction of tumor cells with the microenvironment," Cell Communication and Signaling: CCS, vol. 9, no. 1, p. 18, 2011.

[6] M. A. Scott, J. Shen, K. Lam et al., "Review of pericytes in tumor biology," International Journal of Orthopaedics, vol. 2, no. 3, pp. 300-306, 2015.

[7] M. Crisan, C.-W. Chen, M. Corselli, G. Andriolo, L. Lazzari, and B. Péault, "Perivascular multipotent progenitor cells in human organs," Annals of the New York Academy of Sciences, Hematopoietic Stem Cells VII, vol. 1176, pp. 118-123, 2009.

[8] E. Pierantozzi, M. Badin, B. Vezzani et al., "Human pericytes isolated from adipose tissue have better differentiation abilities than their mesenchymal stem cell counterparts," Cell and Tissue Research, vol. 361, no. 3, pp. 769-778, 2015.

[9] C. Pieper, J. J. Marek, M. Unterberg, T. Schwerdtle, and H. J. Galla, "Brain capillary pericytes contribute to the immune defense in response to cytokines or LPS in vitro," Brain Research, vol. 1550, pp. 1-8, 2014.

[10] B. Gökçinar-Yagci, D. Uçkan-Çetinkaya, and B. Çelebi-Saltik, "Pericytes: properties, functions and applications in tissue engineering," Stem Cell Reviews and Reports, vol. 11, no. 4, pp. 549-559, 2015.

[11] H. Gerhardt and C. Betsholtz, "Endothelial-pericyte interactions in angiogenesis," Cell and Tissue Research, vol. 314, no. 1, pp. 15-23, 2003.

[12] K. D. Barlow, A. M. Sanders, S. Soker, S. Ergun, and L. J. Metheny-Barlow, "Pericytes on the tumor vasculature: jekyll or hyde?" Cancer Microenvironment, vol. 6, no. 1, pp. 1-17, 2013.

[13] P. C. Stapor, R. S. Sweat, D. C. Dashti, A. M. Betancourt, and W. L. Murfee, "Pericyte dynamics during angiogenesis: new insights from new identities," Journal of Vascular Research, vol. 51, no. 3, pp. 163-174, 2014.
[14] Y. Minami, T. Sasaki, J. Kawabe, and Y. Ohsaki, "Accessory cells in tumor angiogenesis-tumor-associated pericytes," in Research Directions in Tumor Angiogenesis, J. Chai, Ed., pp. 73-89, InTech, 2013, https://www.intechopen.com/ books/research-directions-in-tumor-angiogenesis/accessorycells-in-tumor-angiogenesis-tumor-associated-pericytes.

[15] M. Orriols, A. Guadall, M. Galán et al., "Lysyl oxidase (LOX) in vascular remodelling. Insight from a new animal model," Thrombosis and Haemostasis, vol. 112, no. 4, pp. 812824, 2014.

[16] P. Trackman, D. Saxena, and M. Bais, "Two functions of lysyl oxidases: extracellular matrix maturation and cell proliferation," The FASEB Journal, vol. 29, Supplement 1, pp. 570-618, 2015.

[17] P. C. Trackman, "Enzymatic and non-enzymatic functions of the lysyl oxidase family in bone," Matrix Biology, vol. 52-54, pp. 7-18, 2016.

[18] H. M. Kagan and W. Li, "Lysyl oxidase: properties, specificity, and biological roles inside and outside of the cell," Journal of Cellular Biochemistry, vol. 88, no. 4, pp. 660-672, 2003.

[19] K. Csiszar, "Lysyl oxidases: a novel multifunctional amine oxidase family," Progress in Nucleic Acid Research and Molecular Biology, vol. 70, pp. 1-32, 2001.

[20] C. Rodríguez, A. Rodríguez-Sinovasm, and J. MartínezGonzález, "Lysyl oxidase as a potential therapeutic target," Drug News \& Perspectives, vol. 21, no. 4, p. 218, 2008.

[21] H. E. Barker, T. R. Cox, and J. T. Erler, "The rationale for targeting the LOX family in cancer," Nature Reviews Cancer, vol. 12, no. 8, pp. 540-552, 2012.

[22] M. Nilsson, H. Adamo, A. Bergh, and S. Halin Bergström, "Inhibition of lysyl oxidase and lysyl oxidase-like enzymes has tumour-promoting and tumour-suppressing roles in experimental prostate cancer," Scientific Reports, vol. 6, article 19608, 2016.

[23] M. W. Pickup, H. Laklai, I. Acerbi et al., "Stromally derived lysyl oxidase promotes metastasis of transforming growth factor- $\beta$-deficient mouse mammary carcinomas," Cancer Research, vol. 73, no. 17, pp. 5336-5346, 2013.

[24] H. Kasashima, M. Yashiro, H. Kinoshita et al., "Lysyl oxidase-like 2 (LOXL2) from stromal fibroblasts stimulates the progression of gastric cancer," Cancer Letters, vol. 354, no. 2, pp. 438-446, 2014.

[25] M. Nilsson, C. Hägglöf, P. Hammarsten et al., "High lysyl oxidase (LOX) in the non-malignant prostate epithelium predicts a poor outcome in prostate cancer patient managed by watchful waiting," PLoS One, vol. 10, no. 10, article e0140985, 2015.

[26] M. C. Valadares, J. P. Gomes, G. Castello et al., "Human adipose tissue derived pericytes increase life span in Utrn (tm1Ked) Dmd (mdx) /J mice," Stem Cell Reviews, vol. 10, no. 6, pp. 830-840, 2014.

[27] N. M. Vieira, M. Valadares, E. Zucconi et al., "Human adipose-derived mesenchymal stromal cells injected systemically into GRMD dogs without immunosuppression are able to reach the host muscle and express human dystrophin," Cell Transplantation, vol. 21, no. 7, pp. 1407-1417, 2012.

[28] C. Kaid, P. B. G. Silva, B. A. Cortez, C. O. Rodini, P. SemedoKuriki, and O. K. Okamoto, "miR-367 promotes proliferation and stem-like traits in medulloblastoma cells," Cancer Science, vol. 106, no. 9, pp. 1188-1195, 2015. 
[29] X. Yang, S. Li, W. Li et al., "Inactivation of lysyl oxidase by $\beta$-aminopropionitrile inhibits hypoxia-induced invasion and migration of cervical cancer cells," Oncology Reports, vol. 29, no. 2, pp. 541-548, 2013.

[30] E. Eruslanov and S. Kusmartsev, "Identification of ROS using oxidized DCFDA and flow-cytometry," Methods in Molecular Biology, Advanced Protocols in Oxidative Stress II, part I, vol. 594, pp. 57-72, 2010.

[31] H.-C. Chen, "Boyden chamber assay," Methods in Molecular Biology, vol. 294, part II, pp. 15-22, 2005.

[32] M. Dominici, K. Le Blanc, I. Mueller et al., "Minimal criteria for defining multipotent mesenchymal stromal cells. The International Society for Cellular Therapy position statement," Cytotherapy, vol. 8, no. 4, pp. 315-317, 2006.

[33] V. G. Cooke, V. S. LeBleu, D. Keskin et al., "Pericyte depletion results in hypoxia-associated epithelial-to-mesenchymal transition and metastasis mediated by met signaling pathway," Cancer Cell, vol. 21, no. 1, pp. 66-81, 2012.

[34] J. Hong, N. P. Tobin, H. Rundqvist et al., "Role of tumor pericytes in the recruitment of myeloid-derived suppressor cells," Journal of the National Cancer Institute, vol. 107, no. 10, p. djv209, 2015.

[35] D. Keskin, J. Kim, V. G. Cooke et al., "Targeting vascular pericytes in hypoxic tumors increases lung metastasis via angiopoietin-2," Cell Reports, vol. 10, no. 7, pp. 1066$1081,2015$.

[36] A. L. Ribeiro and O. K. Okamoto, "Combined effects of pericytes in the tumor microenvironment," Stem Cells International, vol. 2015, Article ID 868475, p. 8, 2015.

[37] S. Shrivastav, A. Bal, G. Singh, and K. Joshi, "Tumor angiogenesis in breast cancer: pericytes and maturation does not correlate with lymph node metastasis and molecular subtypes," Clinical Breast Cancer, vol. 16, no. 2, pp. 131-138, 2015.

[38] D. Attwell, A. Mishra, C. N. Hall, F. M. O. Farrell, and T. Dalkara, "What is a pericyte?" Journal of Cerebral Blood Flow \& Metabolism, vol. 36, no. 2, pp. 451-455, 2016.

[39] J. Shen, S. Shrestha, Y.-H. Yen et al., "Pericyte antigens in perivascular soft tissue tumors," International Journal of Surgical Pathology, vol. 23, no. 8, pp. 638-648, 2015.

[40] W. B. Stallcup, W.-K. You, K. Kucharova, P. Cejudo-Martin, and F. Yotsumoto, "NG2 proteoglycan-dependent contributions of pericytes and macrophages to brain tumor vascularization and progression," Microcirculation, vol. 23, no. 2, pp. 122-133, 2015.

[41] M. Kamouchi, T. Ago, and T. Kitazono, "Brain pericytes: emerging concepts and functional roles in brain homeostasis," Cellular and Molecular Neurobiology, vol. 31, no. 2, pp. 175193, 2011.

[42] W. E. Thomas, "Brain macrophages: on the role of pericytes and perivascular cells," Brain Research Reviews, vol. 31, no. 1, pp. 42-57, 1999.

[43] E. A. Winkler, R. D. Bell, and B. V. Zlokovic, "Central nervous system pericytes in health and disease," Nature Neuroscience, vol. 14, no. 11, pp. 1398-1405, 2011.

[44] E. M. Caspani, P. H. Crossley, C. Redondo-Garcia, and S. Martinez, "Glioblastoma: a pathogenic crosstalk between tumor cells and pericytes," PLoS One, vol. 9, no. 7, article e101402, 2014.

[45] L. Cheng, Z. Huang, W. Zhou et al., "Glioblastoma stem cells generate vascular pericytes to support vessel function and tumor growth," Cell, vol. 153, no. 1, pp. 139-152, 2013.
[46] K. Hida, N. Maishi, Y. Sakurai, Y. Hida, and H. Harashima, "Heterogeneity of tumor endothelial cells and drug delivery," Advanced Drug Delivery Reviews, vol. 99, part B, pp. 140147, 2016.

[47] T. Marsh, K. Pietras, and S. S. McAllister, "Fibroblasts as architects of cancer pathogenesis," Biochimica et Biophysica Acta, vol. 1832, no. 7, pp. 1070-1078, 2013.

[48] T. Osawa, N. Ohga, K. Akiyama et al., "Lysyl oxidase secreted by tumour endothelial cells promotes angiogenesis and metastasis," British Journal of Cancer, vol. 109, no. 8, pp. 2237-2247, 2013.

[49] K. Szauter and K. Csiszar, "LOXL3 (Lysyl Oxidase-Like 3)," Atlas of Genetics and Cytogenetics in Oncology and Haematology, vol. 13, no. 9, pp. 644-646, 2009.

[50] J. Zhang, R. Yang, Z. Liu et al., "Loss of lysyl oxidase-like 3 causes cleft palate and spinal deformity in mice," Human Molecular Genetics, vol. 24, no. 21, pp. 6174-6185, 2015.

[51] H. E. Barker, D. Bird, G. Lang, and J. T. Erler, "Tumor-secreted LOXL2 activates fibroblasts through FAK signaling," Molecular Cancer Research, vol. 11, no. 11, pp. 1425-1436, 2013.

[52] V. Barry-Hamilton, R. Spangler, D. Marshall et al., "Allosteric inhibition of lysyl oxidase-like-2 impedes the development of a pathologic microenvironment," Nature Medicine, vol. 16, no. 9, pp. 1009-1017, 2010.

[53] S. Zaffryar-Eilot, D. Marshall, T. Voloshin et al., "Lysyl oxidase-like-2 promotes tumour angiogenesis and is a potential therapeutic target in angiogenic tumours," Carcinogenesis, vol. 34, no. 10, pp. 2370-2379, 2013. 

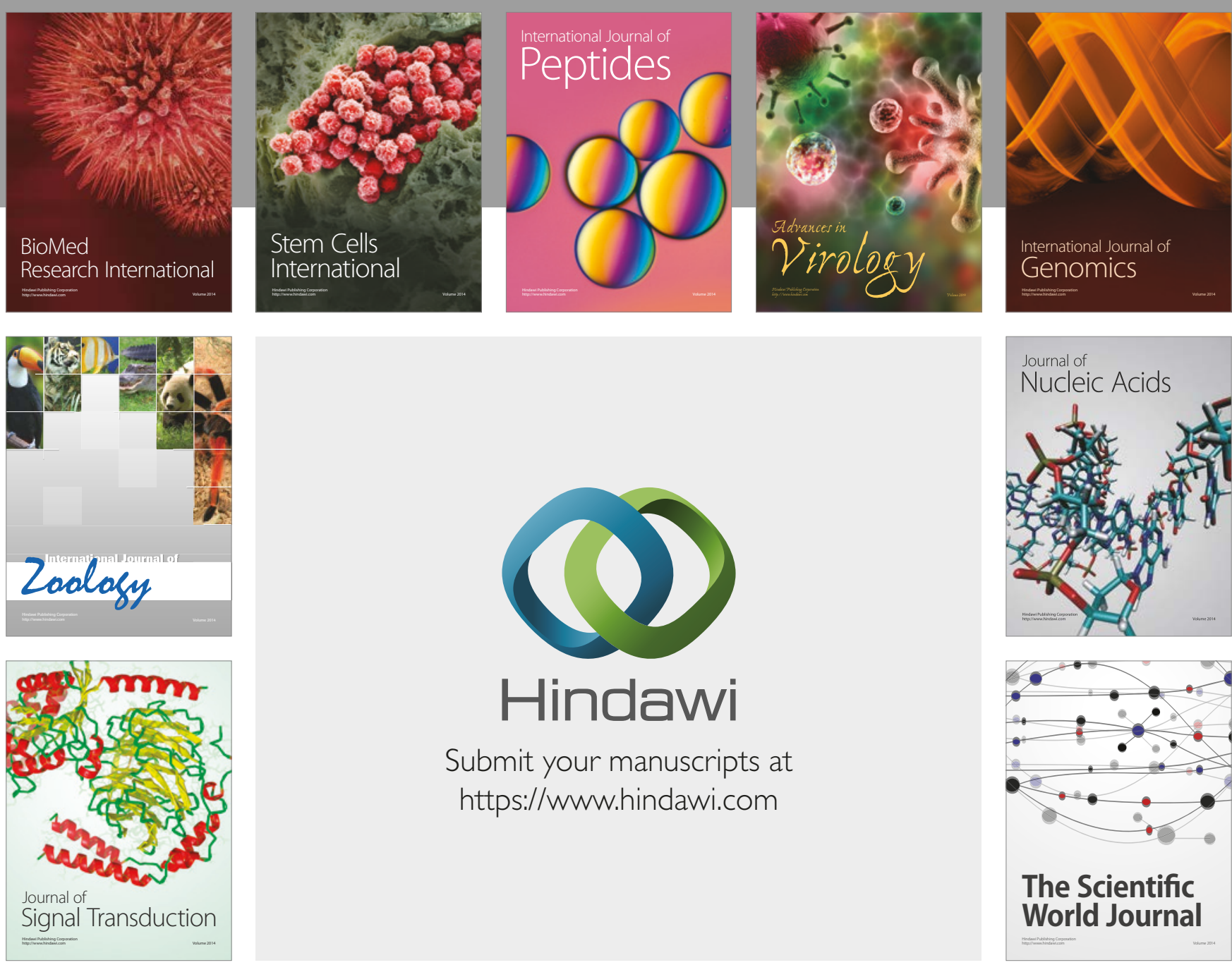

Submit your manuscripts at

https://www.hindawi.com
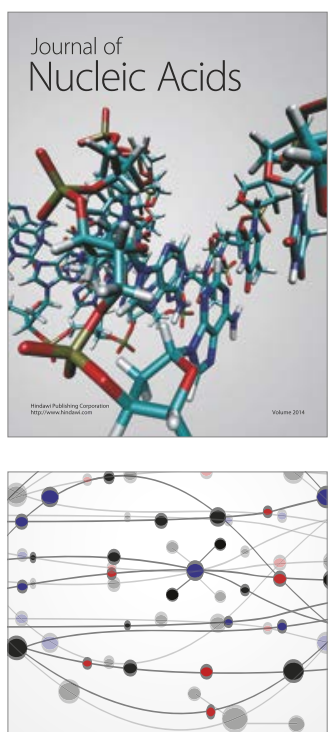

The Scientific World Journal

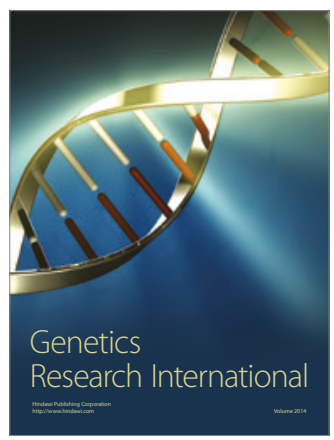

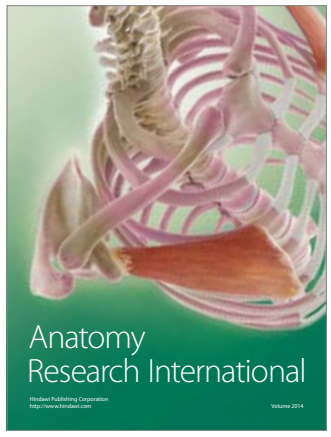

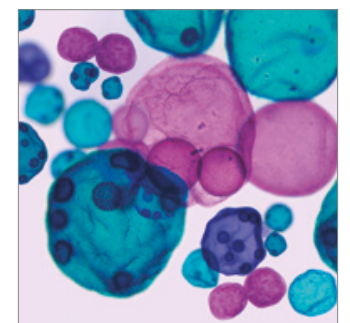

International Journal of Microbiology
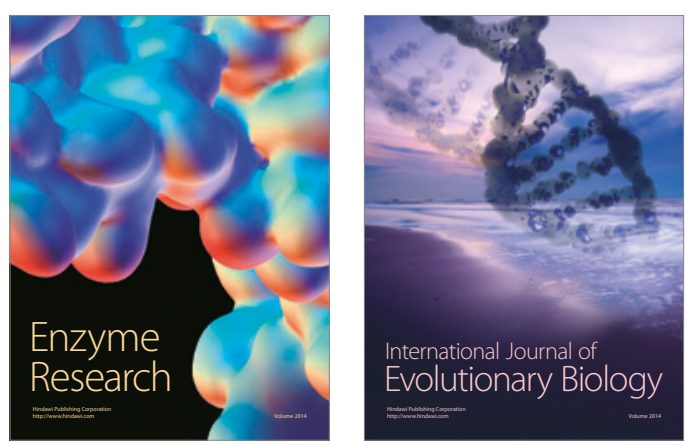
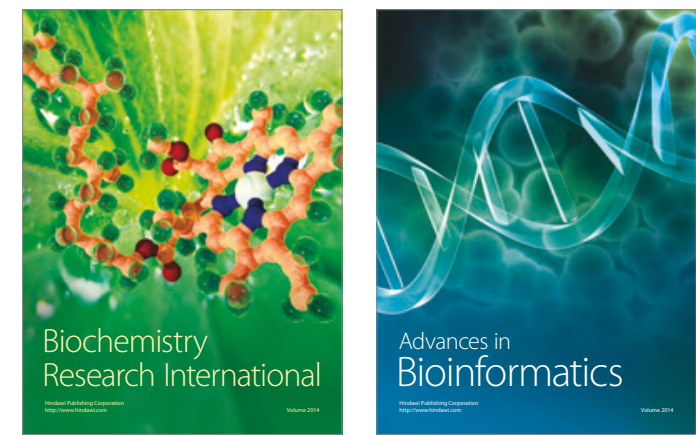

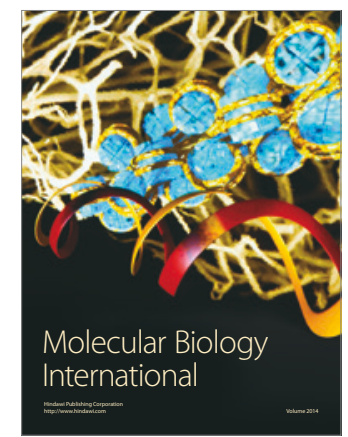

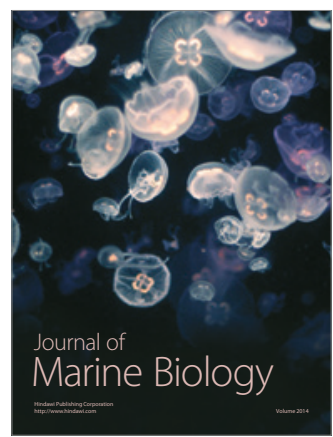

